
\title{
28 Research Square \\ AS-OCT application in quantifying Posterior capsule opacification severity with varied IOL designs
}

\section{SHASHA YU}

Tianjin Eye Hospital https://orcid.org/0000-0001-5336-4519

Chengzhe Lu

Tianjin Eye Hospital

\section{Yawen Guo}

Tianjin Eye Hospital

\section{Yun Zhao}

Tianjin Eye Hospital

Xiaoyong Yuan ( $\nabla$ yuanxy_cn@hotmail.com )

Tianjin Eye Hospital https://orcid.org/0000-0002-0204-8361

\section{Research article}

Keywords: PCO, AS-OCT, IOL-posterior capsule space, IOL-posterior capsule distance, severity

Posted Date: September 4th, 2020

DOI: https://doi.org/10.21203/rs.3.rs-66210/v1

License: (c) (1) This work is licensed under a Creative Commons Attribution 4.0 International License. Read Full License 


\section{Abstract \\ Background}

To evaluate application of AS-OCT in PCO severity assessment and analyse relationship between PCO severity and IOL characters.

\section{Methods}

PCO patients were prospectively recruited. Cross-sectional images of the anterior segment at horizontal and vertical medians were acquired with AS-OCT. The area of the IOL-PC (posterior capsular) space and PCO severity (area, thickness and density at $3 \mathrm{~mm}$ and $5 \mathrm{~mm}$ IOL optic regions) were measured. The relationship between visual acuity and PCO severity, comparisons of PCO severity and IOL-PC space using varied IOL designs were analysed.

\section{Results}

One hundred PCO eyes were enrolled. IOL-PC space and PCO thickness and area was positively correlated with axial length. The cut-off level of visual acuity: was 0.52 Log MAR. Visual acuity were positively correlated with PCO area and thickness when visual acuity was $\leq 0.52$ Log MAR. 3-piece C haptic IOL showed a smaller PCO area and thickness than the 1-piece 3 haptic IOL and 1-piece 4 haptic IOL. PCO area and thickness values for an IOL with a diameter $\leq 11.0 \mathrm{~mm}$ were greater than for an IOL with a diameter of $12.5 \mathrm{~mm}$. The differences were statistically significant. PCO area and thickness increased when IOL haptic angulation increased (from 0 to 12 degrees).

\section{Conclusions}

In PCO eyes, cut-off level of visual acuity was 0.52 Log MAR. With more severe PCO, visual acuity maybe not enough to describe the visual function impairment. PCO severity and IOL-PC space was significantly correlated with axial length and IOL design and material.

\section{Background}

PCO is a major long-term complication of cataract surgery, which normally occurs at several weeks to years after removal of the cloudy lens. The prevalence of PCO is $4.1 \%$ at 1 year and $22.8 \%-38.5 \%$ at $2-$ 4 years [1]. PCO leads to a decrease in visual acuity, contrast sensitivity and objective visual quality. The effect of PCO on visual function should be related to the PCO location, morphology, and severity. PCO severity could be defined in terms of density, coverage, and thickness. 
The existing PCO evaluation methods used to assess PCO include a subjective scoring system, or an objective system, such as the POCO and AQUA systems, which are all based on automated analysis of retro-illumination images. In addition, the Scheimpflug image is an objective method that evaluates the density of the PCO directly. These methods primarily score PCO severity by multiplying the PCO fraction and density, and the latter can be subjectively graded [2]. The methods vary, and a standard method is lacking. Until now, AS-OCT (anterior segment OCT) based on low coherent optical tomography can acquire high-resolution images of the anterior segment [3] and this has been used to evaluate PCO severity based on cross-sectional images of PCO $[4,5]$.

In addition, studies have suggested that surgical factors and the IOL design and material contributed to PCO formation and development [6]. Application of a sharp edged [7], hydrophobic IOL [8] could reduce PCO occurrence [9]. Adherence of the IOL [10], capsule bend formation [11] and capsule crease existence [12] are closely correlated to the formation of PCO. In addition, according to a theory described as no space, no cell and no PCO, the IOL-PC (IOL- posterior capsular) space of an IOL has significant influence on PCO formation. The existence of an IOL-PC space may be related to the size of the capsule, axial length, and IOL design and material, which has not been analysed.

The aim of this study was to evaluate IOL-PC space and PCO severity with AS-OCT (RTVue-100 OCT), including PCO density, PCO coverage (area), and PCO thickness. We also assessed the relation between IOL-PC space and PCO severity and evaluated the effect of the IOL design and material on the IOL-PC space and PCO severity.

\section{Materials And Methods Participants}

Patients diagnosed with PCO and anticipated Nd:YAG laser treatment were prospectively enrolled in our cataract centre, totally 100 eyes. In details, patients with observable PCO under bio-microscope, subjective visual disability symptoms and decreased visual acuity were enrolled. The study strictly adhered to the tenets of the Declaration of Helsinki, and the study was approved by the Research Review Board. Before examination, written informed consent was obtained from each patient. The exclusion criteria included late stage glaucoma, fundus diseases, uveitis, corneal diseases, eye trauma or complicated surgery, IOL tilt and de-centration.

Routine examinations were done by one expert ophthalmologist. Besides, visual acuity, intraocular pressure, slit lamp examination, fundus checking and AS-OCT examination after pupil dilation were performed before Nd:YAG laser treatment.

The visual acuity was valued using a logarithmic visual acuity chart. For further correlation analysis, visual acuity was converted to the logarithm of the minimum angle of resolution (logMAR). 
With reference to medical records, patients were classified into different groups based on the IOL material, haptic design, haptic angulation and IOL diameter. The implanted IOLs included Bausch \& Lomb ADAPT AO (33), ZEISS Bigbag (15), CT ASPHINA 603P ZO (15), Sensar AR40e (14), HQ-201hep (10).

\section{AS-OCT (RTVue-100 OCT) Examinations}

Before RTVue-100 (Optovue Inc., Fremont, CA) image acquisition, a corneal anterior module long adaptor lens was fixed to a detecting probe and software was adjusted to the cornea cross line scan mode. Patients were required to look ahead at the red indicator light with the contralateral eye after pupil dilation. Cross-sectional images of the anterior segment on the horizontal and vertical meridians were acquired after blinking, which were then transferred to a personal computer for further analysis with Image Pro Plus software. The size of the IOL-PC space, IOL-PC distance, and PCO coverage (area), thickness and density at the $3 \mathrm{~mm}$ and $5 \mathrm{~mm}$ IOL optic regions on horizontal and vertical meridians (as described in Fig. 1) were measured and recorded.

We analysed and calculated the mean and median values of the size of the IOL-PC space, IOL-PC distance, and PCO coverage (area), thickness and density. We made comparisons of the IOL-PC space, IOL-PC distance, and PCO area, thickness and density with various IOL designs and materials.

\section{Statistical analysis}

IBM SPSS Statistics 23.0 was adopted to analyse all the data. The data distribution was analysed with a Kolmogorov-Smirnov test. Normally distributed data were described as the mean $\pm S D$, and skewed distributed data were described as the medium and quartile (Q1, Q3). A Kruskal Wallis Test and MannWhitney U non-parametric test were used to compare differences in PCO severity with different IOL material, haptic design, haptic angulation and IOL diameter groups. Pearson's correlation test and the nonparametric Spearman correlation test were used to analyse the relationship between different variables. $P \llbracket 0.05$ was considered statistically significant.

4, to embed secret data, it exploited IPMs in multi-sized Prediction Blocks (PBs) in each Coding Tree Unit (CTU), achieving the improvement in capacity without introducing significant degradation in visual quality while preserving coding efficiency as well. Wang et al. [16] gave an effective IPM-based video steganography for HEVC to maintain rate-distortion optimization and increase empirical security. This algorithm captured the embedding impacts on both neighboring and current PUs by designing a special distortion function, and three-layered isolated channels were established according to the property of IPM coding. Besides, Wang et al. [17] also designed a novel video steganography based on IPM for HEVC, which maintained video quality and improved security performance. It presented the probability distribution of $4 \times 4$ IPMs, and proposed a cover selection rule combined with Coding Units (CUs) and PUs coding information. These steganography methods can maintain good visual quality, which makes it hard to detect the stego-videos by previous steganalysis algorithms.

However, to the best knowledge of the authors, there are few video steganalysis algorithms that can effectively detect IPM-based HEVC steganography, and thus IPM-based steganography algorithms in HEVC lack targeted security detection. Currently, the widely used IPM-based video steganalysis algorithm 
with a better performance was proposed by Zhao et al. [18]. It proposed a calibration-based video steganalysis, and extracted IPM Calibration (IPMC) features to detect the steganography. Although the features were of a low dimension and sensitive to the changes of IPMs, this algorithm was specially designed for H.264/AVC videos, and it did not consider the uniqueness and innovation of HEVC video coding. Therefore, when detecting IPM-based HEVC steganography, the effect will be greatly reduced.

By analyzing modified IPMs are no longer optimal in the existing IPM-based HEVC steganography, we propose a novel IPM steganalysis algorithm based on the Relationship of Adjacent IPMs (RoAIPM) features for HEVC videos. During the process of intra-coding, in order to ensure the quality of compressed videos and minimize the size of bit stream, the IPMs in original videos are usually the optimal IPMs in accordance with the intra-modes coding which defines three Most Probable Modes (MPMs) for current luma PU. However, the IPMs of the modified blocks in such stego-videos are generally not the optimal. In fact, the contents of neighboring image blocks are similar, and thus their optimal IPMs are close. But the characteristic that the IPMs of neighboring blocks are close is destroyed by the embedding of secret information. On the other hand, in HEVC intra prediction process, the MPMs of current luma PU are decided by the IPMs of neighboring encoded luma PBs. Thus, in the stego-videos, the probability that the IPM of current block belongs to the three MPMs is greatly reduced. If we present the IPMs distribution of neighboring blocks and the MPMs of every current block in the form of matrix, and then capture the relationships between the IPM of each embedded block and the adjacent IPMs as well as its MPMs, the steganalysis algorithm will obtain fundamental feature changes of stego-videos. According to this principle, the algorithm captures the RoAIPM features by adopting Gray-Level Co-occurrence Matrix (GLCM), which can reflect the change of the relationship between adjacent IPMs in different directions, so as to achieve the goal of detecting the stego-videos. Although the universality of features from all blocks of different sizes is better, they are less targeted and less sensitive to such steganography, together with the fact that $4 \times 4$ PUs are main covers in all IPM-based steganography, so the RoAIPM features are extracted only from $4 \times 4$ PUs. Finally, the features will be sent into Support Vector Machine (SVM) to classify stego-videos and cover-videos. Experimental results demonstrate that the RoAIPM is sensitive to the modification of IPMs, and the accuracy rates are higher than existing other algorithms. Besides, the proposed algorithm has the lowest computational complexity compared with other works, and thus it is easier to implement.

The rest of this paper is organized as follows: In Sect. 2, the HEVC intra prediction process is introduced, and the general model of IPM-based HEVC steganography is analyzed. In Sect. 3, the producing principle of the RoAIPM features is explained. The proposed steganalysis algorithm is described in detail in Sect. 4. Next, Sect. 5 shows the experimental results and analysis. Finally, the conclusions and future work are given in Sect. 6.

\section{Results}

The study enrolled one hundred eyes (96 patients) diagnosed with PCO, including 36 males (38 eyes) and 60 females (62 eyes). The average age of the patients was $66.98 \pm 10.30$ years (ranging from 37 to 
91 years).

\section{IOL-PC space, IOL-PC distance, PCO area and thickness at the $3 \mathrm{~mm}$ and $5 \mathrm{~mm}$ IOL optic regions}

This study enrolled 100 PCO eyes. The medium size of the IOL-PC space was $0.93 \mathrm{~mm}^{2}$ and the IOL-PC distance was $0.13 \mathrm{~mm}$. PCO coverage was $0.36 \mathrm{~mm}^{2}$, the PCO thickness was $0.12 \mathrm{~mm}$, and the density was 41.93 at the $3 \mathrm{~mm} \mathrm{IOL}$ optic region. At the $5 \mathrm{~mm} \mathrm{IOL}$ optic region, these values were $0.63 \mathrm{~mm}^{2}$, $0.12 \mathrm{~mm}$ and 37.52 , respectively. There were 23 eyes that still showed residual IOL-PC space that was not totally occupied by the LECs and extracellular matrix. The medium size of the IOL-PC space was 1.26 $\mathrm{mm}^{2}$, and the IOP-PC distance was $0.12 \mathrm{~mm}$. PCO coverage, thickness and density were $0.25 \mathrm{~mm}^{2}$, $0.10 \mathrm{~mm}$ and 40.19 at the $3 \mathrm{~mm} \mathrm{IOL}$ optic region and $0.60 \mathrm{~mm}^{2}, 0.11 \mathrm{~mm}$ and 34.81 at the $5 \mathrm{~mm} \mathrm{IOL}$ optic region, respectively. In addition, there were 29 high myopia eyes, which were accompanied by a larger IOL-PC space; the average size of the IOL-PC space was $1.25 \mathrm{~mm}^{2}$, and the IOL-PC distance was $0.17 \mathrm{~mm}$. PCO coverage, thickness and density were $0.44 \mathrm{~mm}^{2}, 0.16 \mathrm{~mm}$ and 39.11 at the $3 \mathrm{~mm}$ IOL optic region and were $0.75 \mathrm{~mm}^{2}, 0.12 \mathrm{~mm}$ and 36.20 at the $5 \mathrm{~mm} \mathrm{IOL} \mathrm{optic} \mathrm{region,} \mathrm{respectively} \mathrm{(as} \mathrm{details}$ described in Table 1 and 2).

Table 1

IOL-PC space and IOL-PC distance of different eyes.

\begin{tabular}{|llll|}
\hline & $\begin{array}{l}\text { All eye } \\
\mathbf{n}=\mathbf{1 0 0}\end{array}$ & $\begin{array}{l}\text { High myopia } \\
\mathbf{n = 2 9}\end{array}$ & $\begin{array}{l}\text { Residual IOL-P space } \\
\text { eye } \mathbf{n}=\mathbf{2 3}\end{array}$ \\
\hline IOL-PC area & $0.93(0.63,1.36)$ & $1.25(0.89,1.76)$ & $1.26(0.83,1.79)$ \\
\hline IOL-PC distance & $0.13(0.10,0.19)$ & $0.17(0.12,0.26)$ & $0.12(0.06,0.14)$ \\
\hline
\end{tabular}


Table 2

PCO area, thickness and density at $3 \mathrm{~mm}$ and $5 \mathrm{~mm} \mathrm{IOL}$ optic region.

$3 \mathrm{~mm}$ IOL optic region M(Q1,Q3)

$5 \mathrm{~mm}$ IOL optic region M(Q1,Q3)

\begin{tabular}{|c|c|c|c|c|c|c|}
\hline PCO & Area & Thickness & Density & Area & Thickness & Density \\
\hline $\begin{array}{l}\text { All eye } n= \\
100\end{array}$ & $\begin{array}{l}0.36 \\
(0.18,0.56)\end{array}$ & $\begin{array}{l}0.12 \\
(0.08,0.18)\end{array}$ & $\begin{array}{l}41.93 \\
(36.07,55.27)\end{array}$ & $\begin{array}{l}0.63 \\
(0.43,0.93)\end{array}$ & $\begin{array}{l}0.12 \\
(0.08,0.15)\end{array}$ & $\begin{array}{l}37.52 \\
(32.00,49.33)\end{array}$ \\
\hline $\begin{array}{l}\text { High } \\
\text { myopia }\end{array}$ & $\begin{array}{l}0.44 \\
(0.34,0.80)\end{array}$ & $\begin{array}{l}0.16 \\
(0.10,0.24)\end{array}$ & $\begin{array}{l}39.11 \\
(33.45,49.87)\end{array}$ & $\begin{array}{l}0.75 \\
(0.58,1.22)\end{array}$ & $\begin{array}{l}0.12 \\
(0.10,0.17)\end{array}$ & $\begin{array}{l}36.20 \\
(28.60,42.40)\end{array}$ \\
\hline \multicolumn{7}{|l|}{$n=29$} \\
\hline $\begin{array}{l}\text { Residual } \\
\text { IOL-PC } \\
\text { space eye } \\
n=23\end{array}$ & $\begin{array}{l}0.25 \\
(0.17,0.41)\end{array}$ & $\begin{array}{l}0.10 \\
(0.07,0.15)\end{array}$ & $\begin{array}{l}40.19 \\
(30.89,50.49)\end{array}$ & $\begin{array}{l}0.60 \\
(0.33,0.76)\end{array}$ & $\begin{array}{l}0.11 \\
(0.08,0.14)\end{array}$ & $\begin{array}{l}34.81 \\
(28.14,47.66)\end{array}$ \\
\hline
\end{tabular}

\section{Relationship between axial length and IOL-PC space, IOP- PC distance, and PCO area and thickness}

Axial length was positively correlated with the size of the IOL-PC space and IOL-PC distance, and the Spearman's correlation coefficients were $0.41(P=0.00)$ and $0.34(P=0.003)$, respectively (as described in Fig. 2a and 2b). Axial length was also positively correlated with PCO area and thickness at the $3 \mathrm{~mm}$ and $5 \mathrm{~mm}$ IOL optic regions. The Spearman's correlation coefficients were $0.34(P=0.002)$ and $0.37(P=$ $0.001)$ at the $3 \mathrm{~mm}$ IOL optic region, and the Spearman's correlation coefficients were $0.32(P=0.005)$ and $0.22(P=0.06)$ at the $5 \mathrm{~mm}$ IOL optic region (as described in Fig. $3 a$ and $3 b)$.

\section{Correlation analysis between visual acuity and PCO severity (PCO area and thickness)}

PCO area and thickness were positively correlated with visual acuity when visual acuity was equal to or smaller than 0.52 Log MAR (as described in Fig. $4 \mathrm{a}$ and $4 \mathrm{~b}$ ). At the $3 \mathrm{~mm}$ IOL optic region, the Spearman's correlation coefficients were $0.30(P=0.03)$ ( $P C O$ area and visual acuity) and $0.27(P=0.049)(P C O$ thickness and visual acuity). At the $5 \mathrm{~mm}$ IOL optic region, the Spearman's correlation coefficients were $0.32(P=0.02)$ ( $P C O$ area and visual acuity) and $0.29(P=0.04)$ ( $P C O$ thickness and visual acuity).

There was no significant correlation between PCO area, thickness and visual acuity when visual acuity was larger than 0.52 Log MAR (as described in Fig. $5 \mathrm{a}$ and $5 \mathrm{~b}$ ). At the $3 \mathrm{~mm}$ IOL optic region, the Spearman's correlation coefficients were $0.01(P=0.93)$ ( $P C O$ area and visual acuity) and $0.26(P=0.10)$ (PCO thickness and visual acuity). At the $5 \mathrm{~mm}$ IOL optic region, the Spearman's correlation coefficients were $0.10(P=0.52)$ (PCO area and visual acuity) and $0.14(P=0.36)(P C O$ thickness and visual acuity).

The cut-off level of visual acuity could be defined as 0.52 Log MAR. 


\section{Comparison of IOL-PC space, IOP-PC distance, and PCO coverage and thickness between different IOL material, haptic design, haptic angulation and IOL diameter groups}

There were 87 eyes that had a medical record to reference and were grouped according to the IOL design and material. Comparisons of details for the different groups are shown in Tables 3 and 4.

Table 3

Comparison of area of IOL-PC space and PCO coverage at $3 \mathrm{~mm}$ and $5 \mathrm{~mm}$ IOL optic region with varied IOL designs.

\begin{tabular}{|c|c|c|c|}
\hline \multirow[t]{2}{*}{ Area } & IOL-PC area & $3 \mathrm{~mm}$ PCO area & $5 \mathrm{~mm}$ PCO area \\
\hline & $M(Q 1, Q 3)$ & $M(Q 1, Q 3)$ & $M(Q 1, Q 3)$ \\
\hline \multicolumn{4}{|l|}{ Haptic } \\
\hline 3 piece $C$ haptic & $0.74(0.42,1.19)$ & $0.29(0.17,0.40)$ & $0.49(0.30,0.70)$ \\
\hline 1 piece 3 haptic & $0.90(0.66,1.46)$ & $0.46(0.32,0.78)$ & $0.76(0.41,1.19)$ \\
\hline 1 piece 4 haptic & $1.08(0.74,1.38)$ & $0.47(0.27,0.69)$ & $0.72(0.54,1.06)$ \\
\hline$P$ & 0.13 & 0.00 & 0.02 \\
\hline \multicolumn{4}{|l|}{ IOL diameter } \\
\hline$\leq 11 \mathrm{~mm}$ & $1.04(0.70,1.38)$ & $0.46(0.27,0.75)$ & $0.75(0.48,1.11)$ \\
\hline$\geq 12.5 \mathrm{~mm}$ & $0.76(0.41,1.21)$ & $0.30(0.17,0.41)$ & $0.50(0.30,0.71)$ \\
\hline$P$ & 0.09 & 0.00 & 0.01 \\
\hline \multicolumn{4}{|l|}{ Haptic angulation } \\
\hline$\otimes 10^{\circ}$ & $0.74(0.39,1.15)$ & $0.31(0.17,0.43)$ & $0.50(0.30,0.72)$ \\
\hline $10^{\circ}$ & $1.06(0.74,1.41)$ & $0.45(0.26,0.71)$ & $0.70(0.48,1.07)$ \\
\hline $12^{\circ}$ & $1.19(0.95,1.68)$ & $0.69(0.43,0.82)$ & $1.03(0.67,1.30)$ \\
\hline$P$ & 0.01 & 0.00 & 0.00 \\
\hline \multicolumn{4}{|l|}{ Material } \\
\hline Hydrophilic & $1.02(0.69,1.37)$ & $0.44(0.27,0.69)$ & $0.72(0.49,1.08)$ \\
\hline Hydrophobic & $0.60(0.34,1.16)$ & $0.20(0.17,0.35)$ & $0.44(0.30,0.61)$ \\
\hline P & 0.05 & 0.00 & 0.01 \\
\hline
\end{tabular}


Table 4

Comparison of distance of IOL-PC space and PCO thickness at $3 \mathrm{~mm}$ and $5 \mathrm{~mm}$ IOL optic region with varied IOL designs.

\begin{tabular}{|c|c|c|c|}
\hline \multirow[t]{2}{*}{ Thickness } & IOL-PC distance & 3mmPCO thickness & $5 \mathrm{mmPCO}$ thickness \\
\hline & M (Q1,Q3) & M (Q1,Q3) & M (Q1,Q3) \\
\hline \multicolumn{4}{|l|}{ Haptic } \\
\hline 3 piece $\mathrm{C}$ haptic & $0.11(0.07,0.13)$ & $0.10(0.07,0.13)$ & $0.08(0.07,0.12)$ \\
\hline 1 piece 3 haptic & $0.16(0.10,0.25)$ & $0.15(0.09,0.23)$ & $0.12(0.08,0.16)$ \\
\hline 1 piece 4 haptic & $0.16(0.11,0.25)$ & $0.15(0.09,0.22)$ & $0.13(0.10,0.17)$ \\
\hline$P$ & 0.00 & 0.01 & 0.00 \\
\hline \multicolumn{4}{|l|}{ IOL diameter } \\
\hline$\leq 11 \mathrm{~mm}$ & $0.16(0.10,0.25)$ & $0.15(0.09,0.21)$ & $0.13(0.09,0.17)$ \\
\hline$\geq 12.5 \mathrm{~mm}$ & $0.11(0.08,0.13)$ & $0.10(0.07,0.13)$ & $0.08(0.07,0.12)$ \\
\hline $\mathrm{P}$ & 0.00 & 0.01 & 0.00 \\
\hline \multicolumn{4}{|l|}{$\begin{array}{l}\text { Haptic } \\
\text { angulation }\end{array}$} \\
\hline$\otimes 10^{\circ}$ & $0.11(0.07,0.13)$ & $0.10(0.07,0.14)$ & $0.09(0.70,0.13)$ \\
\hline $10^{\circ}$ & $0.16(0.10,0.25)$ & $0.12(0.08,0.20)$ & $0.13(0.10,0.17)$ \\
\hline $12^{\circ}$ & $0.23(0.15,0.27)$ & $0.18(0.14,0.25)$ & $0.13(0.09,0.17)$ \\
\hline$P$ & 0.00 & 0.00 & 0.00 \\
\hline \multicolumn{4}{|l|}{ Material } \\
\hline Hydrophilic & $0.15(0.10,0.24)$ & $0.14(0.09,0.20)$ & $0.12(0.08,0.16)$ \\
\hline Hydrophobic & $0.09(0.07,0.12)$ & $0.09(0.06,0.13)$ & $0.09(0.07,0.13)$ \\
\hline P & 0.00 & 0.02 & 0.13 \\
\hline
\end{tabular}

Three-piece $C$ haptic IOLs had a smaller IOL-PC space and IOL-PC distance than 1 piece-3 haptic IOLs and 1 piece-4 haptic IOLs, and the differences in IOL-PC distances were statistically significant $(P=0.001)$. Similar trends could be observed in PCO coverage and thickness results, such that 3-piece $\mathrm{C}$ haptic IOLs had significantly less and thinner PCOs compared with the 1 piece-3 haptic IOLs and 1 piece-4 haptic IOLs (at the $3 \mathrm{~mm}$ IOL optic region, $\mathrm{P}=0.001$ and $\mathrm{P}=0.011$; at the $5 \mathrm{~mm} \mathrm{IOL}$ optic region, $\mathrm{P}=0.016$ and $\mathrm{P}=0.003)$. 
IOL diameters equal to or less than $11.00 \mathrm{~mm}$ had larger IOL-PC spaces $(P=0.089)$ and IOL-PC distances $(P=0.001)$ when compared to IOLs with diameters equal to or larger than $12.5 \mathrm{~mm}$. The IOL diameter equal to or less than $11.00 \mathrm{~mm}$ group showed higher PCO coverage and thickness when compared to the IOL diameters equal to or larger than $12.5 \mathrm{~mm}$ group, and the differences were statistically significant (at the $3 \mathrm{~mm} \mathrm{IOL}$ optic region, $\mathrm{P}=0.001$ and $\mathrm{P}=0.005$; at the $5 \mathrm{~mm} \mathrm{IOL}$ optic region, $\mathrm{P}=0.009$ and $\mathrm{P}=0.003$ ).

The IOL-PC space and distance increased along with an increase in the haptic angulation from 0 degrees to 12 degrees, which were classified into 3 groups. The same trend could be observed in PCO coverage and thickness results. The differences in PCO coverage and thickness between these three groups were significantly different (at the $3 \mathrm{~mm} \mathrm{IOL}$ optic region, $\mathrm{P}=0.00$ and $\mathrm{P}=0.00$; at the $5 \mathrm{~mm} \mathrm{IOL}$ optic region, $P=0.001$ and $P=0.002$ ).

Hydrophilic IOLs showed larger IOL-PC spaces and distances than hydrophobic IOLs, and the differences were statistically significant $(P=0.046$ and $P=0.001)$. Hydrophilic IOLs were also accompanied by more severe PCOs when compared with hydrophobic IOLs (at the $3 \mathrm{~mm} \mathrm{IOL}$ optic region, $\mathrm{P}=0.001$ and $\mathrm{P}=0.021$; at the $5 \mathrm{~mm}$ IOL optic region, $\mathrm{P}=0.005$ and $\mathrm{P}=0.13$ ).

In addition, the PCO density variation was small and had no significant meaning among different groups.

\section{Discussion}

In this study, we first analysed the size of IOL-PC space with cross-sectional anterior segment images that were acquired with RT-Au 100 OCT. It was reported that IOL-PC spaces should be gradually decreased and closed in 2 weeks after IOL implantation $[13,14]$. In our study, we reported the median value of the size of IOL-PC space was $0.93(0.63,1.36) \mathrm{mm}^{2}$ in all these PCO eyes. Besides, it was $1.26(0.83,1.79) \mathrm{mm}^{2}$ in 23 PCO eyes which still showed unoccupied residual IOL-PC space. In high myopia eyes, there was a larger IOL-PC space and more severe PCO. The existence of obvious residual IOL-PC should be closely correlated with PCO formation and severity.

We reported that IOL-PC space was positively correlated with axial length. As reported, capsule space was positively related to axial length, the longer eyes had a larger capsule [6] and the implanted IOL was thinner, which tended to have less convexity of the IOL back surface. In addition, IOL apposition to capsule was negatively correlated with axial length [15]. These factors resulted in a larger IOL-PC space and higher PCO occurrence rate which could be supported by our results as well. In addition, PCO area and thickness were positively correlated with axial length.

The existence of PCO resulted in deceasing of visual acuity. In our study, log MAR visual acuity was positively correlated with PCO area and thickness at the $3 \mathrm{~mm}$ and $5 \mathrm{~mm}$ IOL optic regions when visual

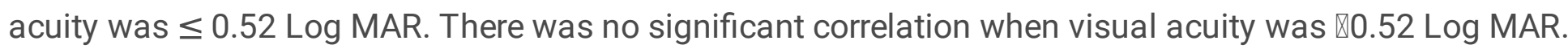
So we believed the cut off level of visual acuity was 0.52 Log MAR in these PCO eyes. These result suggested that the decrease of visual acuity could describe the PCO severity (PCO area and thickness) to some degree. However, with severe PCO more items maybe needed to describe the decreased visual 
function. Besides, In visual acuity $₫ 0.52$ Log MAR group, pear type PCO comprised the major part, the high light scattering of pear type PCO cause more severe visual function impairment, which may influence the results [16]. In previous study, Javier Moreno- Montan e et al. [17] analysed PCO peak density and posterior capsule thickening (PCT) with OCT-1 and reported that log MAR visual acuity was positively correlated with PCT (Spearman correlation coefficient $r=0.66$ ). This agreed with our results. However, Maartje et al. [18] reported a curvilinear correlation between log MAR and PCO severity (EPCO score), the curvilinear correlation indicated that Log MAR is not affected by low PCO severity. This was different from our result. The reason might be the varied PCO severity and different evaluation methods.

We believed that residual IOL-PC and PCO severity could be also attributed to the varied IOL designs and materials. As reported, acrylic, silicone, and poly-methyl methacrylate IOLs showed various capsule closure times, the delayed closure of capsule could facilitate the migration and accumulation of LECs (lens epithelia cells). [11]. As it was reported that the rapid proliferation speed of LECs for patients younger than 40 years was only $5.8 \pm 0.6$ days, for those who were older than 60 years was $7.2 \pm$ 0.7 days. Hence, theoretically, LECs could migrate to and proliferate on the posterior capsule before capsular bend formation (1 month after surgery [11]) and IOL apposition to a capsule (within 2 weeks for normal eyes [13, 14] and en-longed for high myopia eyes [15]). In addition, Elgohary et al. [19] reported closer apposition between IOL optic and mid-peripheral posterior capsules when comparing hydrophobic acrylic IOLs and PH silicone IOLs. When this apposition was missing with a PH silicone IOL, there was high reflectance material that accumulated between the $\mathrm{IOL}$ and posterior capsule, which was considered as LECs and extracellular matrix. In our study, we not only observed these materials but also quantified these high reflectance materials (including PCO area, thickness and density) in the IOP-PC space and proved that there was a positive relation between the size of the IOL-PC space and PCO severity (PCO area and thickness) with RT-Au 100 OCT.

In addition, in these PCO eyes recruited in our study, we analysed the correlation between PCO severity and IOL designs and materials.

In our study, the 1 piece IOL had a larger IOL-PC space and PCO area and thickness than the 3 piece IOL, this might be due to poor axial stability and efficiency of IOL adherence, which was agreed with a previous study [20]. Further more, haptic junction points interrupted the 360-degree sharp edge that hampered capsule bend formation, weakened the mechanical barrier effect of the IOL optic edge [21], and the high multi-crease occurrences [22] which may be the channel for LEC migrations $[8,12]$.

We also found more severe PCOs with hydrophilic IOLs than hydrophobic IOLs. This was agreed with the higher PCO occurrences, PCO scores and Nd:YAG laser surgery rates in previous hydrophilic IOLs related studies $[1,15]$. This might be due to the surface of hydrophilic IOLs, which was more suitable for migration and proliferation of LECs [23]. While, the surface of hydrophobic IOLs showed stiffer adherence with the capsule, and the fibronectin and laminin strengthened the adherence to eliminate the IOL-PC space [10]. In addition, there was higher capsule crease occurrence in hydrophilic IOL eyes which facilitated the LEC migration. 
It was believed that IOLs suited to the capsule could prevent a PCO, ACO, and IOL tile and decentration [24]. In our study, when the implanted IOL diameter was $\leq 11.00 \mathrm{~mm}$, the IOL-PC space and PCO area and thickness increased compared with the IOL diameter $\geq 12.5 \mathrm{~mm}$ group. This might be due to poor IOL adherence and multi-capsule creases. It was reported that the diameter of the empty capsule for emmetropia eyes was $10 \mathrm{~mm}-10.8 \mathrm{~mm}$ [6], when the IOL implanted capsule diameter was $10 \mathrm{~mm}$ or could be stretched to $12.0 \mathrm{~mm}$ [25]. With an IOL diameter $\geq 12.5 \mathrm{~mm}$, the capsule could be stretched to eliminate creases and IOL-PC space. Meanwhile, the powerful mechanical stress of the IOL optic edge prevented the migration of LECs. However, with an IOL diameter increase, PCO may increase. As Javier Moreno-Montan e's et al. [4] reported, when the IOL diameter was $\leq 12.0 \mathrm{~mm}, 12.5 \mathrm{~mm}$, or $\geq 13.0 \mathrm{~mm}$, PCTs were $0 \mu \mathrm{m}$ (0-37), $56 \mu \mathrm{m}$ (17.33-78.66), and $78.66 \mu \mathrm{m}$ (12-133.33), respectively. A thicker PCO in the $\geq 13.0 \mathrm{~mm}$ group might be due to over stretching and could have caused more creases on the haptic axial, which induced the migration of LECs. However, a study also reported that there was no effect of the IOL diameter on PCO formation [6, 26]. Georgios [26] compared Corneal A501D IOLs and Corneal J501D IOLs with different IOL diameters $(11.0 \mathrm{~mm}$ and $10.5 \pm 0.1 \mathrm{~mm})$, and the PCO AQUA scores were $2.13 \pm$ 1.64 and $2.0 \pm 1.74$, respectively. Efstathios Vounotrypidis [6] reported no significant difference in PCO EPCO scores between Quatrix ${ }^{\circledR} I O L(11.0 \mathrm{~mm})$ and Quatrix Evolutive ${ }^{\circledR} \mathrm{IOL}(10.47 \pm 0.13 \mathrm{~mm}, 10.3-$ $10.8 \mathrm{~mm}$ ). The difference between these results and ours might be due to the smaller IOL diameter differences in Georgios' and Efstathios Vounotrypidis's studies.

We also found that PCO area and thickness were increased along with the increases in IOL haptic angulation. This agreed with Javier Moreno-Montan $e^{\prime}$ s [4] results, which were IOL haptic angulation of 0,5 , and 10 degrees when the PCTs were $0 \mathrm{~mm}$ (0-37.33), $58.67 \mathrm{~mm}$ (17.33-92.00), and $59.33 \mathrm{~mm}(0.67-$ 110.50), respectively, which were significantly different. It was reported that AcrySof $\AA$ MA60BM with 10degree haptic angulation showed axial movement at 3 months after surgery. The poor axial stability hampered the closing of the capsule, which may promote PCO formation. However, it was believed that haptic angulation could increase the contact between the IOL and capsule and that 10-degree haptic angulation was preferable [27]. Some others also suggested that there was no influence of IOL haptic angulation on PCO formation. Schmidbauer [28] implanted Centerflex ${ }^{\circledR}$ IOLs (Rayner) with 0, 5, and 10 degree haptic angulations in rabbit eyes and reported no significant differences in the central PCO, peripheral PCO and Soemmering's ring scores. The author believed that the mechanical stress of the IOL edge may overwhelm the effect of haptic angulation.

\section{Conclusions}

In conclusion, RT-Au 100 OCT can be applied to objectively analyse and quantify the apposition between an IOL and the posterior capsule and PCO severity (area, thickness and density). It was suggested that IOL-PC space and PCO area and thickness were positively correlated with axial length. In addition, PCO area and thickness was positively correlated with visual acuity. The cut off level of visual acuity was 0.52 Log MAR. when visual acuity was larger than 0.52 Log MAR,more items may be needed to describe the visual function impairment. Besides, IOL-PC space and PCO area and thickness were also well related 
with IOL designs. 3-piece $\mathrm{C}$ haptic, diameter of $12.5 \mathrm{~mm}$, smaller haptic angulation and hydrophobic IOL tended to have smaller IOL-PC space and less severe PCO.

\section{Abbreviations}

IOLs: intraocular lens; AS-OCT: anterior segment Optical coherence tomography; IOL-PC: IOL- posterior capsular space; PCO: posterior capsule opacification; Nd:YAG: Nd Yttrium Aluminum Garnet; LECs: lens epithelia cells; PCT: posterior capsule thickening, logMAR: Logarithm of the minimum angle of resolution; ACO: anterior capsule opacification

\section{Declarations}

\section{Acknowledgements}

Doctors in Cataract Center who provided and cared the study patients and doctors in Cataract Center and Ocular Function Examination Area who provided technical help should be acknowledged.

\section{Funding}

There was no specific funding of this study.

\section{Availability of data and materials}

The datasets analyzed during the current study are available from the corresponding author on reasonable request.

\section{Authors' contributions}

SSY and CZL designed this study. SSY and YZ collected and analyzed all the data. SSY and CZL drafted and revised the work. XYY and YWG give suggestion and direction during all the study. SSY, CZL, YWG, $Y Z$ and $X Y Y$ substantively revised the manuscript. All authors read and approved the final manuscript.

\section{Ethics approval and consent to participate}

The study was reviewed and approved by the Research Review Broad of Tianjin Eye Hospital. Informed consent of paper edition from each patient was collected before the examination and surgery.

\section{Consent for publication}

Not Applicable.

\section{Competing interests}

The authors declare that they have no competing interests. 


\section{References}

1. Kossack N, Schindler C, Weinhold I, Hickstein L, Lehne M, Walker J, et al. German claims data analysis to assess impact of different intraocular lenses on posterior capsule opacification and related healthcare costs. Z Gesundh Wiss. 2018;26:81-90.

2. Buehl W, Findl O. Effect of intraocular lens design on posterior capsule opacification. J Cataract Refract Surg. 2008;34:1976-85.

3. Hagag AM, Gao SS, Jia Y, Huang D. Optical coherence tomography angiography: Technical principles and clinical applications in ophthalmology. Taiwan J Ophthalmol. 2017;7:115-29.

4. Moreno-Montanes J, Alvarez A, Bes-Rastrollo M, Garcia-Layana A. Optical coherence tomography evaluation of posterior capsule opacification related to intraocular lens design. J Cataract Refract Surg. 2008;34:643-50.

5. Yu S, Lu C, Tang X, Yuan X, Yuan B, Yu Z Application of Spectral Domain Optical Coherence Tomography to Objectively Evaluate Posterior Capsular Opacity In Vivo. Journal of Ophthalmology, 2018, 2018:5461784.

6. Vounotrypidis E, Lackerbauer C, Kook D, Dirisamer M, Priglinger S, Mayer WJ. Influence of total intraocular lens diameter on efficacy and safety for in the bag cataract surgery. Oman $\mathrm{J}$ Ophthalmol. 2018;11:144-9.

7. Haripriya A, Chang DF, Vijayakumar B, Niraj A, Shekhar M, Tanpreet S, et al. Long-term Posterior Capsule Opacification Reduction with Square-Edge Polymethylmethacrylate Intraocular Lens: Randomized Controlled Study. OPHTHALMOLOGY. 2017;124:295-302.

8. Schriefl SM, Leydolt C, Stifter E, Menapace R. Posterior capsular opacification and Nd:YAG capsulotomy rates with the iMics Y-60H and Micro AY intra-ocular lenses: 3-year results of a randomized clinical trial. ACTA OPHTHALMOL. 2015;93:342-7.

9. Perez-Vives C. Biomaterial Influence on Intraocular Lens Performance: An Overview. J OPHTHALMOL. 2018;2018:2687385.

10. Oshika T, Nagata T, Ishii Y. Adhesion of lens capsule to intraocular lenses of polymethylmethacrylate, silicone, and acrylic foldable materials: an experimental study. Br J Ophthalmol. 1998;82:549-53.

11. Nishi O, Nishi K, Akura J. Speed of capsular bend formation at the optic edge of acrylic, silicone, and poly(methyl methacrylate) lenses. J Cataract Refract Surg. 2002;28:431-7.

12. Nishi O, Nishi K. Effect of the optic size of a single-piece acrylic intraocular lens on posterior capsule opacification. J Cataract Refract Surg. 2003;29:348-53.

13. Tao A, Lu P, Li J, Shao Y, Wang J, Shen M, et al. High resolution OCT quantitative analysis of the space between the IOL and the posterior capsule during the early cataract postoperative period. Invest Ophthalmol Vis Sci. 2013;54:6991-7.

14. Hayashi H, Hayashi K, Nakao F, Hayashi F. Elapsed time for capsular apposition to intraocular lens after cataract surgery. OPHTHALMOLOGY. 2002;109:1427-31. 
15. Zhao Y, Yang K, Li J, Huang Y, Zhu S. Comparison of hydrophobic and hydrophilic intraocular lens in preventing posterior capsule opacification after cataract surgery: An updated meta-analysis. Medicine. 2017;96:e8301.

16. Lu C, Yu S, Song H, Zhao Y, Xie S, Tang X, Yuan X. Posterior capsular opacification comparison between morphology and objective visual function. BMC Ophthalmology, 2019;19;1;40.

17. Moreno-Montanes J, Alvarez A, Maldonado MJ. Objective quantification of posterior capsule opacification after cataract surgery, with optical coherence tomography. Invest Ophthalmol Vis Sci. 2005;46:3999-4006.

18. van Bree MC, van den Berg TJ, Zijlmans BL. Posterior capsule opacification severity, assessed with straylight measurement, as main indicator of early visual function deterioration. OPHTHALMOLOGY. 2013;120:20-33.

19. Elgohary MA, Chauhan DS, Dowler JG. Optical coherence tomography of intraocular lens implants and their relationship to the posterior capsule: a pilot study comparing a hydrophobic acrylic to a plate-haptic silicone type. OPHTHALMIC RES. 2006;38:116-24.

20. Fong CS, Mitchell P, Rochtchina E, Cugati S, Hong T, Wang JJ. Three-year incidence and factors associated with posterior capsule opacification after cataract surgery: The Australian Prospective Cataract Surgery and Age-related Macular Degeneration Study. AM J OPHTHALMOL. 2014;157:1719.

21. Sugita M, Kato S, Sugita G, Oshika T. Migration of lens epithelial cells through haptic root of singlepiece acrylic-foldable intraocular lens. AM J OPHTHALMOL. 2004;137:377-9.

22. Shah GD, Vasavada AR, Praveen MR, Shah AR, Trivedi RH. Incidence and influence of posterior capsule striae on the development of posterior capsule opacification after 1-piece hydrophobic acrylic intraocular lens implantation. J Cataract Refract Surg. 2012;38:202-7.

23. Dorey MW, Brownstein S, Hill VE, Mathew B, Botton G, Kertes PJ, et al. Proposed pathogenesis for the delayed postoperative opacification of the hydroview hydrogel intraocular lens. AM J OPHTHALMOL. 2003;135:591-8.

24. Lim SJ, Kang SJ, Kim HB, Apple DJ. Ideal size of an intraocular lens for capsular bag fixation. J Cataract Refract Surg. 1998;24:397-402.

25. Assia El, Legler UF, Apple DJ. The capsular bag after short- and long-term fixation of intraocular lenses. OPHTHALMOLOGY. 1995;102:1151-7.

26. Mylonas G, Georgopoulos M, Prinz A, Vock L, Blum RA, Schmidt-Erfurth U. Influence of a variable overall diameter hydrophilic acrylic sharp-edged single-piece intra-ocular lens on capsule opacification one year after surgery. CURR EYE RES. 2014;39:620-5.

27. WesendahI TA, Hunold W, Auffarth GU, Apple DJ. [Area of contact of the artificial lens and posterior capsule. Systematic study of various haptic parameters]. OPHTHALMOLOGE. 1994;91:680-4.

28. Schmidbauer JM, Escobar-Gomez M, Apple DJ, Peng Q, Arthur SN, Vargas LG. Effect of haptic angulation on posterior capsule opacification in modern foldable lenses with a square, truncated optic edge. J Cataract Refract Surg. 2002;28:1251-5. 


\section{Figures}

\section{Anterior surface of $10 \mathrm{~L}$}

A

B

C.

D

\section{1, Posterior surface of $10 \mathrm{~L}$}

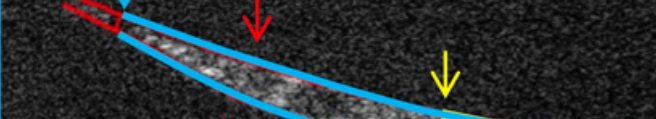

Posterior capsule

\section{Figure 1}

Illustration graph of PCO severity evaluation method with RTVue-100 OCT PCO severity evaluates in one cross-sectional image. Point $C$ is the center of the (red, horizontal) line that passed optic center of IOL. Distance between point $A$ and point $E$ is the $5 \mathrm{~mm}$ range of $\mathrm{IOL}$ optic region. Distance between point $\mathrm{B}$ and point $D$ is the $3 \mathrm{~mm}$ range of IOL optic region. The crescent-shaped region between posterior surface and posterior capsule is the IOP-PC space. The region between the yellow lines is the PCO coverage at 3 $\mathrm{mm}$ range of the $\mathrm{IOL}$ optic region. The region between the blue lines is the PCO coverage at $5 \mathrm{~mm}$ range of the IOL optic region. The yellow and blue arrows refer to the PCO thickness at the specific points, including the $3 \mathrm{~mm}$ and $5 \mathrm{~mm}$ points of the $\mathrm{IOL}$ optic region.
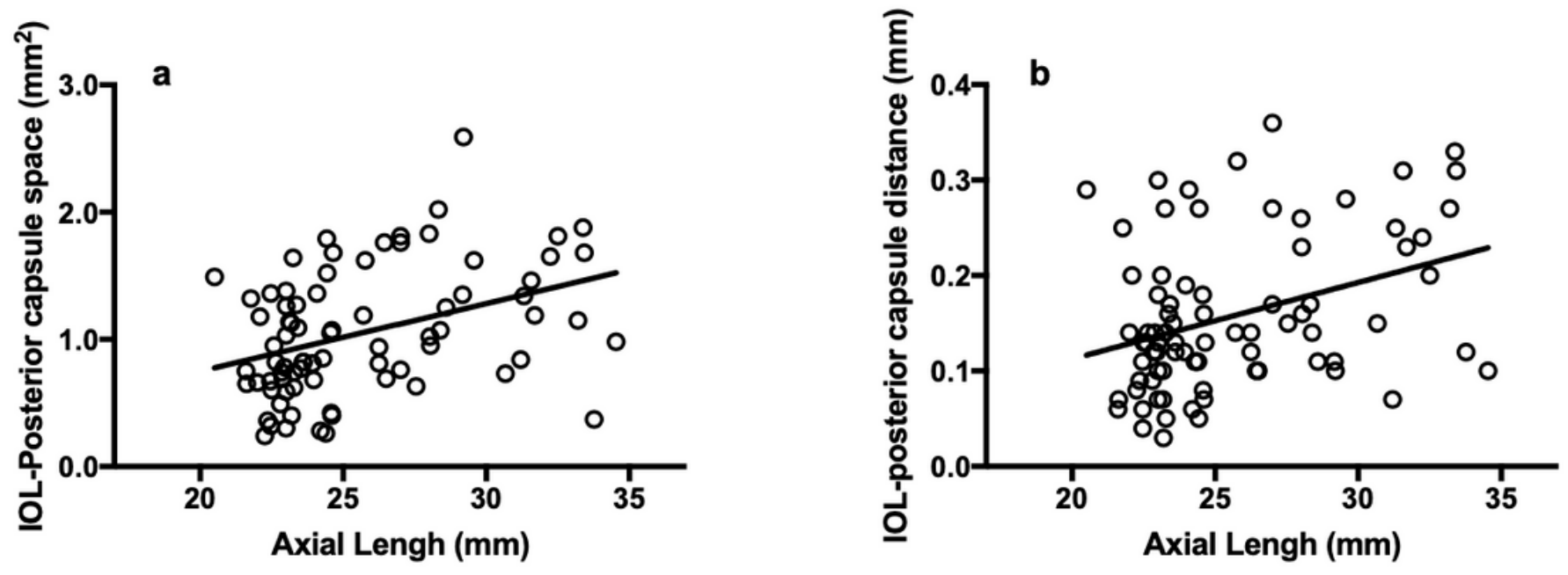

Figure 2 
Relationship between axial length and IOL-PC space area and distance. a) Correlation between axial length and IOL-PC area, the correlation coefficient is $0.41, P=0.00$. b) Correlation between axial length and IOL-PC distance, the correlation coefficient is $0.34, \mathrm{P}=0.00$
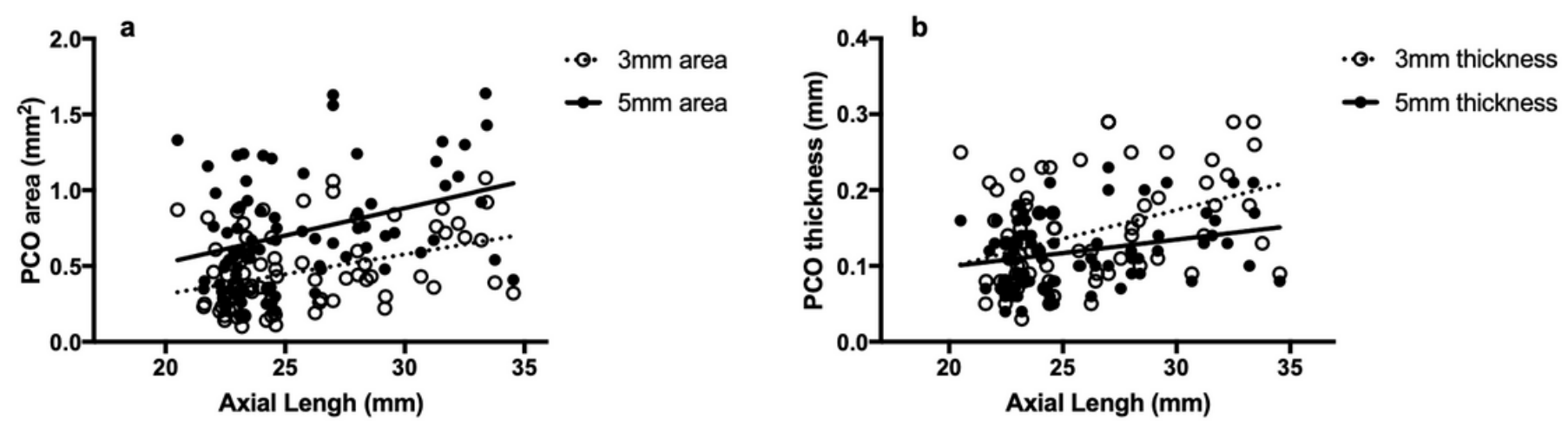

\section{Figure 3}

Relationship between axial length and PCO area and thickness. a) Correlation between axial length and $\mathrm{PCO}$ area at $3 \mathrm{~mm}$ and $5 \mathrm{~mm}$ IOL optic region, the correlation coefficient is $0.34(\mathrm{P}=0.00)$ and 0.32 $(P=0.01)$. b) Correlation between axial length and $P C O$ thickness at $3 \mathrm{~mm}$ and $5 \mathrm{~mm}$ IOL optic region, the correlation coefficient is $0.37(P=0.001)$ and $0.22(P=0.06)$.
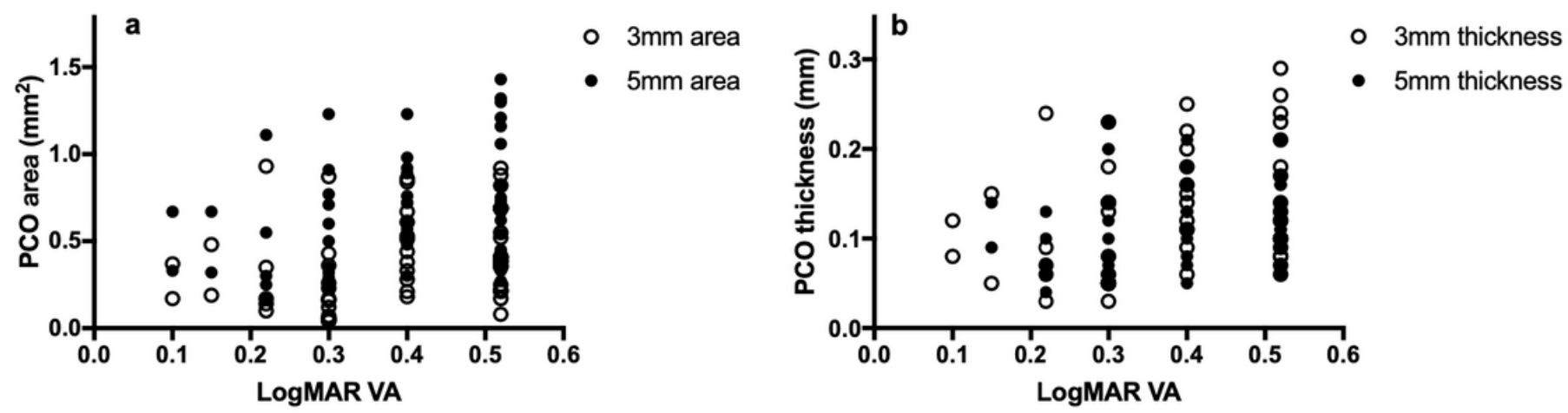

Figure 4

Relationship between visual acuity and PCO area and thickness when visual acuity is $\geq 0.3$. a) Correlation of visual acuity \& PCO area at $3 \mathrm{~mm}$ and $5 \mathrm{~mm}$ IOL optic region, the correlation coefficient is and $0.30(P=0.03)$ and $0.32(P=0.02)$, respectively. b) Correlation of visual acuity \& $P C O$ thickness at $3 \mathrm{~mm}$ and $5 \mathrm{~mm}$ IOL optic region, the correlation coefficient is $0.27(P=0.049)$ and $0.29(P=0.04)$, respectively. 

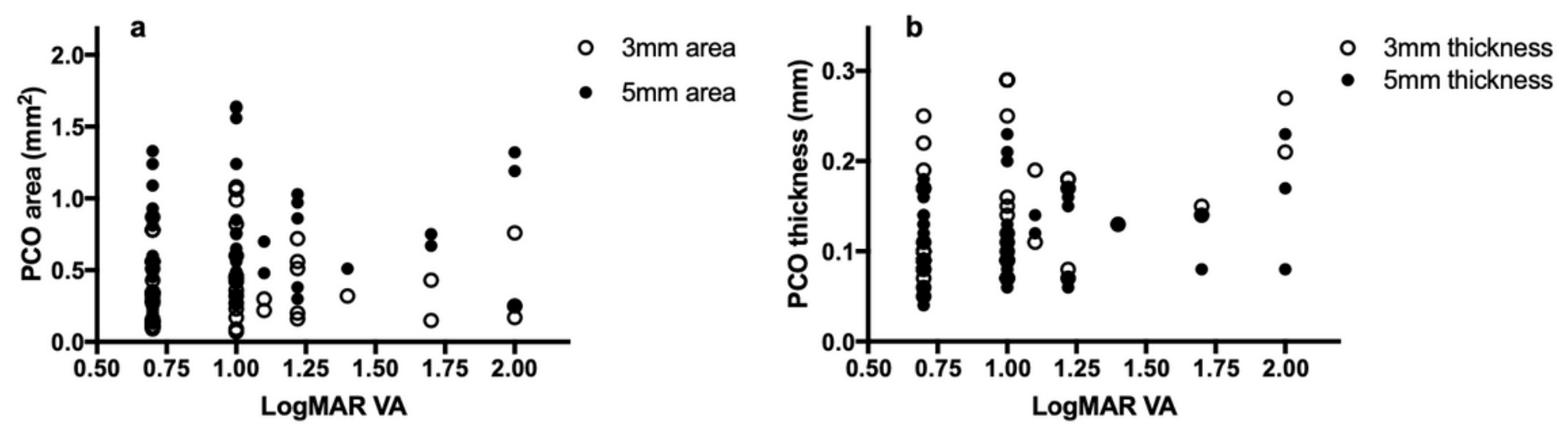

Figure 5

Relationship between visual acuity and PCO area and thickness when visual acuity is $₫ 0.3$. a) Correlation of visual acuity \& PCO area at $3 \mathrm{~mm}$ and $5 \mathrm{~mm}$ IOL optic region, the correlation coefficient is $0.01(P=0.93)$ and $0.10(P=0.52)$, respectively. b) Correlation of visual acuity \& PCO thickness at $3 \mathrm{~mm}$ and $5 \mathrm{~mm} \mathrm{IOL}$ optic region, the correlation coefficient is $0.26(P=0.10)$ and $0.14(P=0.36)$, respectively. 